\title{
Representations of a compact group on a Banach space.
}

\author{
By Kôji SHIGA
}

(Received Jan. 14, 1955)

\section{Introduction.}

Let $G$ be a compact group and $\Re$ a complex normed linear space. We mean by a representation $\{\Re, T(a)\}$ a homomorphism of $G$ into the group of bounded linear operators on $\mathfrak{R}$ such that $a \rightarrow T(a)$. The following types of representations are important: i) algebraic representation, ii) bounded algebraic representation, ${ }^{1)}$ iii) weakly measurable representation, ${ }^{2}$ iv) strongly measurable representation, ${ }^{3)}$ v) weakly continuous representation, ${ }^{4)}$ vi) strongly continuous representation. ${ }^{5)}$ Clearly each condition above becomes gradually stronger when it runs from i) to vi), except iv). When the representation space $\mathfrak{R}$ of $\{\mathfrak{R}, T(a)\}$ is a Banach space, it is called a B-representation.

In considering a B-representation $\{\mathfrak{B}, T(a)\}$, what we shall call the conjugate representation $\left\{\mathfrak{B}^{*}, T^{*}(a)\right\}$ arouses our special interest. For example, we can prove, by considering $\left\{\mathfrak{B}^{* *}, T^{* *}(a)\right\}$ of a weakly continuous B-representation $\{\mathfrak{B}, T(a)\}$, the equivalence of all four conditions (Theorem 2) : 1) $\{\mathfrak{B}, T(a)\}$ is completely decomposable, 2) $\{\mathfrak{B}$, $T(a)\}$ is strongly continuous, 3$)\{\mathfrak{B}, T(a)\}$ is strongly measurable, 4) for any given $x \in \mathfrak{B}$, the closed invariant subspace generated by $T(a) x$,

1) Algebraic representation such that $\|T(a)\|<M$ for a certain $M(>0)$.

2) Bounded algebraic representation such that $f(T(a) x)$ is a measurable function on $G$ for any $x \in \mathfrak{N}$ and $f \in \mathfrak{R}^{*}$, where $\mathfrak{R}^{*}$ means the conjugate space of $\mathfrak{N}$.

3) Bounded algebraic representation such that $T(a) x$ is strongly measurable (in the sense of Bochner) for any fixed $x \in \mathfrak{\Re}$.

4) Bounded algebraic representation such that $f(T(a) x)$ is a continuous function on $G$ for any $x \in \mathfrak{N}$ and $f \in \mathfrak{N}^{*}$.

5) Algebraic representation such that

(and then necessarily bounded).

$$
\left\|T\left(a_{\alpha}\right) x-T\left(a_{0}\right) x\right\| \rightarrow 0 \quad \text { as } \quad a_{\alpha} \rightarrow a_{0},
$$


$a \in G$, is separable. This explains naturally the classical result of Peter and Weyl [10].'

The content of the present paper is briefly as follows. We first give a survey of unitary representations of a compact group (§1). In $\S 2$ the finite dimensionality of an irreducible algebraic representation is proved under a weak assumption. Our discussions which follow are really based on two fundamental Propositions A and B $(\S 3)$; in fact, Proposition A yields the essential part of the structure Theorem 2 $(\S 4)$. On the other hand, it is possible to deduce from Proposition B a formal structure of weakly continuous representations which might be regarded as a formulation of reduction of the weak continuity to the strong one $(\S 5)$. The relations between the weak and the strong continuity are discussed in $\$ 6$. The rôle played by the weak and the strong measurable representations becomes clear in the last section $(\S 7)$. The principal idea used here is to reduce an arbitrary representation to a unitary one. Since we make full use of the boundedness of Haar measure, it seems difficult to generalize further this method beyond the compact case.

Throughout this paper we concern ourselves with the representation of a compact group $G$. $\mathfrak{g}, \mathfrak{B}$ and $\mathfrak{R}$ denote a Hilbert space, a Banach space and a normed linear space, respectively; Hilbert space is not assumed to be separable. Linear operators are assumed to be bounded and are denoted by $S(a), T(a), U(a)$ etc., whose adjoint operators are denoted by $S^{*}(a), T^{*}(a)$ etc. respectively. We denote elements of a compact group $G$ by $a, b, c, \cdots$, and elements of $\mathfrak{N}, \mathfrak{B}, \mathfrak{S}$ etc, by $x, y, z, \cdots ; f, g, \cdots$. The integral is always taken with respect to the Haar measure on $G . L^{p}(G)(1 \leqq p \leqq \infty)$ and $C(G)$ (the family of continuous functions on $G$ ) denote the usual Banach spaces. For a given $\mathfrak{N}$, we shall denote by $[x \mid P(x)]$ the closed subspace of $\mathfrak{N}$, generated by all elements $x$ having the property $P(x)$.

The author wishes to express his deepest thanks to Professors Y. Mimura and Y. Kawada for their constant encouragements and advices during the preparation of this paper. The author is also grateful to Mr. N. Iwahori for his valuable remarks.

6) Numbers in brackets refer to the bibliography at the end of the paper. 


\section{§1. Unitary representations.}

In this section we consider a unitary representation $\{\mathfrak{S}, U(a)\}$. The following three propositions are well-known." Since Proposition 1 plays a fundamental rôle for our later discussions, we give here a direct proof of Proposition 1.

PROPOSITION 1. $\{\mathfrak{S}, U(a)\}$ is completely reducible, i.e. $\mathfrak{S}$ is a discrete direct sum of mutually orthogonal finite-dimensional subspaces $\mathfrak{S}_{\infty}$ 's, each of which is invariant and irreducible under $U(a)(a \in G)$ : $\mathfrak{H}=\sum_{\alpha} \oplus \mathfrak{H}_{\alpha}$.

PROOF. Since the invariance of a closed subspace of $\mathfrak{S}$ implies that of its orthogonal complement, it suffices to prove this proposition only in the case where $\mathfrak{E}$ is generated by $U(a) \xi(a \in G)$ for some fixed $\xi,\|\xi\|=1$.

For all $x, y$ in $\mathfrak{s}$, we define

$$
[x, y]=\int(x, U(a) \xi) \overline{(y, U(a) \xi)} d a .
$$

Normalizing Haar measure so that $\int d a=1$, we have $[x, y] \leqq\|x\|\|y\|$. Hence $[x, y]$ defines a bounded bilinear form on $\mathfrak{k}$. Let $H$ be the Hermitian operator associated with $[x, y]^{8)}$ :

$$
[x, y]=(H x, y) \quad \text { for all } x, y \text {. }
$$

Then $H$ is a positive definite operator in the strict sense. Now, for any $a_{0} \in G$ and for every $x, y$ in $\mathfrak{S}$, we have

$$
\begin{aligned}
\left(U\left(a_{0}\right) H x, y\right) & =\left(H x, U\left(a_{0}\right)^{*} y\right) \\
& =\int(x, U(a) \xi) \overline{\left(y, U\left(a_{0} a\right) \xi\right)} d a \\
& =\int\left(U\left(a_{0}\right) x, U(a) \xi\right)(y, U(a) \xi) d a \\
& =\left(H U\left(a_{0}\right) x, y\right),
\end{aligned}
$$

whence each $U(a)$ commutes with $H$. Moreover, $H$ is completely

7) See Godement [1], Hurevitsch [5], Itô [6], Murakami [8]] and Wigner [14].

8) This Hermitian operator $H$ was first introduced explicitly by S. Itô [6]. 
continuous. In fact, if $x_{n} \rightarrow x_{0}$ (weakly), $H x_{n} \rightarrow H x_{0}$ (weakly); on the other hand, from (1) and Lebesgue's convergence theorem follows that $\left\|H x_{n}\right\|^{2} \rightarrow\left\|H x_{0}\right\|^{2}$, and thus $H x_{n} \rightarrow H x_{0}$ (strongly).

Consequently, if we perform the spectral resolution of $H$, we have $H=\sum \lambda_{i} P_{\mathfrak{M i} i}$, where $P_{\mathfrak{M i} i}$ is the projection whose range is the finitedimensional eigenspace $\mathfrak{M}_{i}$ corresponding to the eigenvalue $\lambda_{i}$. This, together with the positive definiteness of $H$ in the strict sense, yields

$$
\mathfrak{S}=\sum \oplus \mathfrak{M}_{\boldsymbol{i}} \text {. }
$$

Since each finite-dimensional subspace $\mathfrak{M}_{i}$ reduces every $U(a)$, because of the commutativity of $H$ and $U(a)$, the above decomposition of $\mathfrak{S}$ gives a decomposition into invariant subspaces. If $\left\{\mathfrak{M}_{i} U_{\mathfrak{M}_{i}}(a)\right\}^{9)}$ is still reducible, it is possible further to decompose $\mathfrak{M}_{i}$ into irreducible subspaces by the use of the well-known procedures. This completes the proof.

We call such a decomposition of $\mathfrak{S}$ as stated in Proposition 1 a complete decomposition of $\{\mathfrak{H}, U(a)\}$. Now we shall establish the relationship between two complete decompositions of $\{\mathfrak{F}, U(a)\}$. This is easily formulated in terms of the following notion: For a complete decomposition $\left\{\mathfrak{S}_{\mathfrak{S}}, U(a)\right\}=\sum_{a} \oplus\left\{\mathfrak{E}_{\alpha}, U_{\mathfrak{h}_{\alpha}}(a)\right\}$ and for a fixed equivalent class $\mathfrak{D}$ of irreducible representations of $G$, we denote by $\mathfrak{S}(\mathfrak{D})$ the closed subspace generated by all those $\mathfrak{S}_{\alpha}$ 's for which the irreducible representation $U_{\mathfrak{L}_{\alpha}}(a)$ belongs to $\mathfrak{D}$. Thus, $\mathfrak{S}=\sum_{\alpha} \oplus \mathfrak{S}_{\alpha}$ and $\mathfrak{S}=\sum_{\beta} \oplus \mathfrak{F}_{\beta}$ denoting two complete decompositions of $\{\mathfrak{F}, U(a)\}$, they yield another kind of decompositions such that $\mathfrak{S}=\sum_{\mathfrak{D}} \oplus \mathfrak{S}(\mathfrak{D})$ and $\mathfrak{L}=\sum_{\mathfrak{D}} \oplus \mathfrak{S}^{\prime}(\mathfrak{D})$ respectively, with respect to the class of irreducible representations. Then from the orthogonality relations we get the following result.

PROPOSITION 2. $\mathfrak{S}(\mathfrak{D})=\mathfrak{S E}^{\prime}(\mathfrak{D})$.

Proposition 3. Any irreducible unitary representation of a compact group is equivalent to one which is obtained in a complete decomposition of the regular representation.

Proof. Let $\{\mathfrak{S}, U(a)\}$ be irreducible. Then making use of notations in the proof of Proposition 1, we have $H=\lambda I(\lambda>0)$, that is

9) $U_{\mathfrak{W}_{i}}(a)$ denotes the representation of $G$ obtained by restricting $U(a)$ in $\mathfrak{M}_{i}$. Such notation is used in $\S 1$; in the remaining sections, if it happens such a case, we write $U(a)$ for $U_{\mathfrak{m}_{i}}(a)$ only for simplicity. 


$$
\lambda\|x\|^{2}=\int|(x, U(a) \xi)|^{2} d a .
$$

Hence if we assign to every $x$ of $\mathfrak{g}$ an element $f_{x}(a)=(x, U(a) \xi) / \sqrt{\lambda}$ of $L^{2}(G)$, we obtain an isomorphism between $\mathfrak{r}$ and a closed subspace $\widetilde{L}$ of $L^{2}(G)$, through which $U(a)$ can be identified with the regular representation in $\widetilde{L}$.

REMARK. This result can be generalized to a theorem for locally compact groups which asserts that any irreducible unitary representation corresponding to an integrable positive definite function (in Godement's sense [1]] is realizable in the regular representation.

\section{\$2. Irreducible representations. ${ }^{10)}$}

In his paper [7] I. Kaplansky remarked the fact without giving its proof that any irreducible strongly continuous B-representation is finitedimensional. It is a purpose of this section to formulate this theorem in a more generalized form and to prove it.

THEOREM 1. Let $\{\mathfrak{R}, T(a)\}$ be an irreducible bounded algebraic representation of $G$. If for some non-zero element $f_{0} \in \mathfrak{R}^{*} f_{0}(T(a) x)$ is continuous on $G$ for any $x \in \mathfrak{R}$, then $\mathfrak{R}$ is finite-dimensional.

Proof. Set

$$
(x, y)=\int f_{0}(T(a) x) \overline{f_{0}(T(a) y)} d a
$$

for $x, y \in \Re$. From the assumption the integrand being continuous, this definition is meaningful. We shall prove that $\left(x_{0}, x_{0}\right)=0$ implies $x_{0}=0$. In fact, $\left(x_{0}, x_{0}\right)=0$ means

$$
\int\left|f_{0}\left(T(a) x_{0}\right)\right|^{2} d a=0,
$$

and thus $f_{0}\left(T(a) x_{0}\right)=T^{*}(a) f_{0}\left(x_{0}\right)=0$ for all $a \in G$; accordingly, putting $\Re_{0}=\left\{x ; T^{*}(a) f_{0}(x)=0\right.$ for all $\left.a \in G\right\}$, we have $x_{0} \in \Re_{0}$. But $\Re_{0}$ is clearly an invariant closed subspace, whence from the irreducibility of $\{\Re$, $T(a)\}$ and $f_{0} \neq 0$ it results $\mathfrak{R}_{0}=\{0\}$, and so $x_{0}=0$ as desired. $(x, y)$ defined in (2) therefore gives an inner product of $\mathfrak{N}$. 5 denotes the completion of $\mathfrak{R}$ with respect to this inner product and III III denotes

10) Irreducibility of a representation means the non-existence of a proper invariant closed subspace. 
the norm in $\mathfrak{S}$. If $x_{n} \rightarrow x_{0}$ (strongly), we have $T^{*}(a) f_{0}\left(x_{n}\right) \rightarrow T^{*}(a) f_{0}\left(x_{0}\right)$ for each $a$, and so

$$
\left\|\left|x_{n}-x_{0} \|\right|^{2}=\int\left|f_{0}\left(T(a) x_{n}\right)-f_{0}\left(T(a) x_{0}\right)\right|^{2} d a \rightarrow 0 ;\right.
$$

hence $\mathfrak{R}$ is continuosly imbedded into $\mathfrak{S}$ in the sense of the strong topology. We denote the image of $\mathfrak{N}$ into $\mathfrak{S}$ by $\mathfrak{S}(\mathfrak{R})$.

Now consider $T(a)$ as an operator on $\mathfrak{g}(\mathfrak{R})$. We have by the right invariance of Haar measure

$$
\text { II } T\left(a_{0}\right) x\left\|\left.\right|^{2}=\int\left|f_{0}\left(T\left(a a_{0}\right) x\right)\right|^{2} d x=\left|\|x \mid\|^{2} .\right.\right.
$$

Thus, being isometric, $T(a)$ can be uniquely extended to a unitary operator $U(a)$ on $\mathfrak{g}$, which clearly induces an algebraic representation of $G$. We assert furthermore that $\{\mathfrak{S}, U(a)\}$ is a unitary representation of $G$. This follows immediately from the fact that the function $f_{0}(T(b a) x) f_{0}(T(b) y)$ is continuous on $G \times G$.

Applying Proposition 1 to $\{\hat{s}, U(a)\}$, we obtain a complete decom. position of $\mathfrak{S}$ :

$$
\mathfrak{S}=\sum_{\alpha} \oplus \mathfrak{S}_{\alpha}=\sum_{\alpha} \oplus P_{\alpha} \mathfrak{S},
$$

where $P_{a}$ means the projection operator associated with $\mathfrak{g}_{\alpha}$.

Suppose that there is a non-zero $x$ in $\mathscr{S}(\mathfrak{R})$ whose projection on some $\mathfrak{S}_{\alpha_{0}}$ is zero, i. e. $x \neq 0$ and $P_{\alpha_{0}} x=0$. Putting $\mathfrak{S}_{1}=\mathfrak{D} \Theta \mathfrak{S}_{\alpha_{0}}$, this assumption is equivalent to $\mathfrak{I}(\mathfrak{R}) \cap \mathfrak{S}_{1} \neq\{0\}$. Let $\mathfrak{N}_{1}$ be the inverse image of $\mathfrak{S}(\mathfrak{R}) \cap \mathfrak{S}_{1}$ through the natural mapping of $\mathfrak{R}$ onto $\mathfrak{E}(\mathfrak{R})$. Since this mapping is continuous, $\mathfrak{R}_{1}$ is a closed subspace of $\Re$ different from $\{0\}$; mereover, the invariance of $\mathfrak{S}_{1}$ under $U(a)$ implies that of $\mathfrak{R}_{1}$ under $T(a)$. Thus the irreducibility of $\{\mathfrak{R}, T(a)\}$ leads to $\mathfrak{R}_{1}=\mathfrak{R}$. But this means $\mathfrak{S}(\mathfrak{R})=\mathfrak{S}_{\mathfrak{C}}(\mathfrak{R}) \cap \mathfrak{S}_{1}$, and so $\mathfrak{g}(\mathfrak{R}) \subseteq \mathfrak{L}_{1}$, which is contradictory to the fact that $\mathfrak{S}(\mathfrak{R})$ is dense in $\mathfrak{S}$.

Hence, from $x \in \mathfrak{S}(\mathfrak{R})$ and $x \neq 0$, we can conclude $P_{\alpha} x \neq 0$ for all $\alpha$. Taking any $\alpha_{0}$, let $x$ correspond to $P_{\alpha_{0}} x$. Then this induces an isomorphic mapping of $\mathfrak{R}$ into $\mathfrak{F}_{\alpha_{0}}$ regarded as linear spaces. Now $\mathfrak{S}_{\alpha_{0}}$ being finite-dimensional, we find that $\mathfrak{R}$ is also finite-dimensional. This completes the proof.

After accomplishing the proof, observe that the finite dimensionality of $\mathfrak{R}$ implies $\mathfrak{S}=\mathfrak{S}(\mathfrak{R})$. This shows that the complete decomposition of 
$\{\mathfrak{S}, U(a)\}$ given above is trivial.

REMARK. In Theorem 1, if $\mathfrak{N}$ is furthermore assumed to be separable, the assumption on the continuity of $f_{0}(T(a) x)$ can be replaced by the measurability. This is shown by the use of the proof of Theorem 9.

\section{§3. Main propositions.}

Suggested by the result of Peter and Weyl, which clarifies the structure of the regular B-representation induced in $C(G)$, we introduce

DEFINITION 1. For a given algebraic representation $\{\mathfrak{N}, T(a)\}$, let $\left\{\mathfrak{N}_{\alpha}, T(a)\right\}_{\alpha \in A}$ be the family of all the finite-dimensional irreducible representations such that $\mathfrak{N}_{\alpha}$ 's are invariant subspaces of $\mathfrak{N} . \quad\{\mathfrak{l}, T(a)\}$ (or $\mathfrak{i}$ ) is called completely-decomposable if the subspace generated by all $\left\{\mathfrak{N}_{\alpha}\right\}_{\alpha \in A}$ is dense in $\mathfrak{X}$ with respect to the strong topology.

From Theorem 1 follows that, if $\{\mathfrak{N}, T(a)\}$ is weakly continuous, the assumption on finite dimensionality of $\mathfrak{l}_{\alpha}$ in the above definition becomes superfluous.

Now we shall prove two fundamental Propositions A and B, which seem to be, in a sense, in a dual relation. These propositions are very similar to each other not only in the statement but also in the proof. However, as is seen later, they possess in an essential feature the different significance for the theory of representations and will be used rather independently.

PROPOSITION A. Let $\{\mathfrak{i}, T(a)\}$ be a bounded algebraic representation of $G$, and let $\{\subseteq, T(a)\}$ be a weakly continuous representation such that $\mathfrak{S}$ is an invariant subspace of $\mathfrak{l}_{.}^{11)}$ Then, regarding $\left\{\mathfrak{N}^{* *}\right.$, $\left.T^{* *}(a)\right\}$ as a bounded algebraic representation of $G$, we can find an invariant closed subspace $\widetilde{\subseteq}$ of $\mathfrak{M}^{* *}$ with the following properties.

i) $\left\{\widetilde{S}, T^{* *}(a)\right\}$ is strongly continuous and completely decomposable.

ii) Let an element $f$ of $\mathfrak{N}^{*}$ have the property: $F(f)=0$ for all $F \in \widetilde{\mathfrak{S}}$. Then $f$ belongs to $\mathfrak{S}^{+}$, where $\mathfrak{S}^{+}=[f \mid f(x)=0$ for all $x \in \mathfrak{S}]$.

PRoof. Taking any fixed $\xi \in \mathbb{S}$, we define an inner product in $\mathfrak{R}^{*}$ by

$$
(f, g)_{\xi}=\int f(T(a) \xi) g(T(a) \xi) d a
$$

11) In such a case, we say that $\{\subseteq, T(a)\}$ is contained in $\{\mathfrak{R}, T(a)\}$. 
Let $\mathfrak{R}_{\xi}^{*}$ be the closed subspace of $\mathfrak{R}^{*}$ formed by all elements $f$ with $(f, f)_{\xi}=0$; we obtain a Hilbert space $\mathfrak{s}_{\xi}$ by completion of the quotent space $\mathfrak{R}^{*} / \mathfrak{N}_{\xi}^{*}$. Denote the canonical mapping of $\mathfrak{R}^{*}$ into $\mathfrak{S}_{\xi}$, by $f \rightarrow \dot{f}$, and the imbedded image of $\mathfrak{N}^{*}$ into $\mathfrak{S}_{\xi}$, by $\mathfrak{S}_{\xi}\left(\mathfrak{N}^{*}\right)$. Since the representation $\{\Re, T(a)\}$ is bounded, we may assume by (3)

$$
\|\dot{f}\|_{\xi} \leqq\|f\|,
$$

where $\mid\|\| \|_{\xi}$ means the norm of $\mathfrak{S}_{\xi}$. Now, from $T^{*}(a) \Re_{\xi}^{*} \sqsubseteq \Re_{\xi}^{*}$, it follows that $T^{*}(a)$ induces an operator in $\mathfrak{S}_{\xi}\left(\mathfrak{N}^{*}\right)$; this operator being isometric by (3), we can extend it to a unitary operator $U^{*}(a)$ on $\mathfrak{b}_{\xi}$. Remembering the proof of Theorem 1, we know that $\left\{\mathfrak{g}_{\xi}, U(a)\right\}$ thus obtained is a unitary representation of $G$, and that $\mathfrak{b}_{\xi}$ admits a complete decomposition with respect to this representation such that

$$
\mathfrak{S}_{\xi}=\sum_{\infty} \oplus \mathfrak{S}_{\xi_{0} \infty} .
$$

Consider the linear functional $F_{\xi, h}(f)$ on $\mathfrak{N}^{*}$ associated with each element $h \in \mathfrak{S}_{\xi}$, defined by $F_{\xi . h}(f)=(\mathfrak{f}, h)_{\xi}$. We have by (4)

$$
\left|F_{\xi_{s},}(f)\right| \leqq\|f\| \cdot\|h\|_{\xi},
$$

hence $F_{\xi, h}(f)$ defines a bounded linear functional on $\mathfrak{R}^{*}$. If $h_{1} \neq h_{2}$, we have $\left(\dot{f}, h_{1}\right)_{\xi} \neq\left(\dot{f}, h_{2}\right)_{\xi}$ for at least one $\dot{f}$, i.e. $F_{\xi, h_{1}} \neq F_{\xi, h_{2}}$. From (5) follows $\left\|F_{\xi, h}\right\| \leqq\|h \mid\|_{\xi}$.

Put

$$
\mathfrak{N}_{\xi}^{* *}=\left[F_{\xi, h} \mid h \in \mathfrak{S}_{\xi}\right] ;
$$

$\mathfrak{N}_{\xi}^{* *}$ is a closed subspace of $\mathfrak{R}^{* *}$. Denote by $\Psi_{\xi}$ the mapping of $\mathfrak{S}_{\xi}$ into $\Re_{\xi}^{* *}$ such that $h \rightarrow F_{\xi, h}$. Then we have shown that $\Psi_{\xi}$ induces a univalent anti-homomorphism of $\mathfrak{h}_{\xi}$ into $\mathfrak{N}_{\xi}^{* *}$, which is continuous with respect to the strong topology in each space.

Now observe that the operator $U(a)$ on $\mathfrak{S}_{\xi}$ is transferred by $\Psi_{\xi}$ to such an operator on $\mathfrak{R}_{\xi}^{* *}$ as the one obtained by restricting $T^{* *}(a)$ to $\mathfrak{N}_{\xi}^{* *}$; in fact,

$$
\begin{aligned}
F_{\xi, U(a) h}(f) & =(\dot{f}, U(a) h)_{\xi}=\left(U^{*}(a) \dot{f}, h\right)_{\xi} \\
& =F_{\xi, h}\left(T^{*}(a) f\right)=T^{* *}(a) F_{\xi, h}(f) .
\end{aligned}
$$


From this it follows that $\Psi_{\xi}\left(s_{\xi, \alpha}\right)$ is an irreducible invariant finitedimensional subspace in $\left\{\mathfrak{H}^{* *}, T^{* *}(a)\right\}$, and that $\left\{\Psi_{\xi}\left(\mathfrak{s}_{\xi, a}\right), T^{* *}(a)\right\}$ is strongly continuous. $\Psi_{\xi}$ being continuous, the subspace generated by all $\Psi_{\xi}\left(\mathfrak{w}_{\xi, \alpha}\right)$ is dense in $\Re_{\xi}^{* *}$, whence $\left\{\mathfrak{S}_{\xi}^{* *}, T^{* *}(a)\right\}$ is strongly continuous and completely decomposable, as is readily verified.

Finally, put

$$
\widetilde{S}=\left[\Re_{\xi}^{* *} \mid \xi \in \subseteq\right] \text {. }
$$

We show that $\widetilde{\Xi}$ meets the requirements i) and ii) stated in the proposition.

i) follows from the fact that $\widetilde{\subseteq}$ is generated by the closed subspaces $\Re_{\xi}^{* *}$ in which $T^{* *}(a)$ is strongly continuous.

ii) is proved as follows: Choose an $f$ in $\mathfrak{l}^{*}$ such that $F(f)=0$ for all $F \in \widetilde{\mathcal{S}}$. If $f_{\notin} \Im^{+}$, we have $f\left(x_{0}\right) \neq 0$ for an $x_{0} \in S$. Consider $F_{x_{0}, \dot{f}}$; then we have $F_{x_{0}, \dot{f}}(f)=0$ and so

$$
\int\left|f\left(T(a) x_{0}\right)\right|^{2} d a=0,
$$

which implies $f\left(x_{0}\right)=0$ by the continuity of $f\left(T(a) x_{0}\right)$. This contradiction shows the validity of ii).

Thus the proof of Proposition A is completed.

Before we enter into Proposition B, for a given bounded algebraic representation $\{\mathfrak{l}, T(a)\}$ it is convenient to introduce the subspace $\mathfrak{N}_{\mathcal{C}}^{*}$ of $\mathfrak{i}^{*}$ defined by

$$
\mathfrak{R}_{C}^{*}=\{f ; f(T(a) x) \text { is continuous on } G \text { for any } x\} .
$$

Clearly $\mathfrak{M}_{C}^{*}$ is a closed subspace which is invariant under $T^{*}(a)$.

Proposition B. Let $\{\mathfrak{H}, T(a)\}$ be a bounded algebraic representation. Regarding $\left\{\mathfrak{H}^{*}, T^{*}(a)\right\}$ as a bounded algebraic anti-representation, we can find an invariant closed subspace $\widetilde{\mathbb{L}}$ of $\mathfrak{M}^{*}$ with the following properties.

i) $\left\{\widetilde{\mathfrak{Z}}, T^{*}(a)\right\}$ is strongly continuous and completely decomposable.

ii) Let an element $x$ of $\mathfrak{i}$ have the property: $f(x)=0$ for all $f \in \widetilde{\mathfrak{R}}$. Then $x$ belongs to $\mathfrak{\Re}_{c}^{*+}$, where $\mathfrak{\Re}_{c}^{*-\mathcal{L}}=\left[x \mid f(x)=0\right.$ for all $\left.f \in \mathfrak{R}_{c}^{*}\right]$. PROOF. Although almost all parts of this proof go on simply by a slight modification, or rather simplification of the proof of Proposition A, we shall follow it briefly for the sake of later use. 
For any fixed $f \in \mathfrak{R}_{C}^{*}$ we define an inner product in $\mathfrak{R}$ by

$$
(x, y)_{f}=\int f(T(a) x) \overline{f(T(a) y)} d a .
$$

Denoting by $\mathfrak{R}_{f}$ the closed subspace of $\mathfrak{N}$ consisting of all elements $(x, x)_{f}=0$, we obtain a Hilbert space $\mathfrak{S}_{f}$ by completion of $\Re / \Re_{f}$. Consider a unitary representation $\left\{\mathfrak{g}_{f}, U(a)\right\}$ induced naturally from $\left\{\mathfrak{N}, T_{(}(a)\right\}$. Then $\mathfrak{S}_{f}$ is decomposed such that

$$
\mathfrak{S}_{f}=\sum_{\alpha} \oplus \mathfrak{S}_{f, a} \text {. }
$$

For any $z \in \mathfrak{S}_{f}$, the linear functional $\Phi_{f, z}$ on $\mathfrak{R}$ defined by

$$
\Phi_{f, z}(x)=(\dot{x}, z)
$$

belongs to $\mathfrak{R}^{*}$, and the anti-linear mapping $\Psi_{f}$ of $\mathfrak{h}_{f}$ into $\mathfrak{R}^{*}$ such that $z \rightarrow \Phi_{f, z}$ is univalent and continuous. Denote by $\mathfrak{R}_{f, \alpha}^{*}$ the image of $\mathfrak{S}_{f, a}$ mapped by $\Psi_{f}$. Then $\mathfrak{N}_{f, \alpha}^{*}$ is a finite-dimensional irreducible invariant subspace of $\mathfrak{N}^{*}$ with respcet to $T^{*}(a)$; furthermore the representation $\left\{\mathfrak{N}_{f, \alpha}^{*}, T^{*}(a)\right\}$ is strongly continuous. Thus, as is readily seen, putting

$$
\widetilde{\mathfrak{L}}=\left[\Phi_{f, z} \mid z \in \mathfrak{S}_{\mathfrak{e}}, f \in \mathfrak{N}_{C}^{*}\right],
$$

we have the desired closed subspace, and this completes the proof.

It is clear that $\widetilde{\mathfrak{L}}$ possesses the subspace generated by all elements $\Phi_{f, y}$ of $\mathfrak{R}^{*}$ as a dense subspace, where

$$
\Phi_{f, y}(x)=\int f(T(a) x) \overline{f(T(a) y)} d a \quad\left(y \in \Re, f \in \mathfrak{N}_{C}^{*}\right) .
$$

This fact becomes essential in $\S 5$.

\section{\$4. Structure of strongly continuous B-representations.}

In order to explain our aim in this section, let us consider Proposition A. This suggests: if a weakly continuous representation $\{\Re, T(a)\}$ is given, we have a strongly continuous representation which is contained in $\left\{\Re^{* *}, T^{* *}(a)\right\}$. This representation space is rather thickly contained in $\mathfrak{l}^{* *}$, but not always in $\mathfrak{N}$ if we regard $\mathfrak{i}$ as a subspace of $\mathfrak{R}^{* *}$. While this situation will be pursued in $\S 6$, we first examine 
a case where such a strongly continuous representation is already realized in $\{\mathfrak{N}, T(a)\}$. Conditions to guarantee us such a normal case lead naturally to a strongly continuous B-representation, and there we meet the theorem of Peter-Weyl.

THEOREM 2. Let $\{\mathfrak{B}, T(a)\}$ be a weakly continuous B-representation. Then the four properties listed below ar: equivalent to each other:

1) $\{\mathfrak{B}, T(a)\}$ is completely decomposable.

2) $\{\mathfrak{B}, T(a)\}$ is strongly continuous.

3) $\{\mathfrak{B}, T(a)\}$ is strongly measurable.

4) For every $x \in \mathfrak{B}$, the invariant closed subspace $[T(a) x \mid a \in G]$ is separable. ${ }^{12)}$

Proof. We shall prove this theorem by showing a sequence of implications : 1$) \rightarrow 2) \rightarrow 4) \rightarrow 3) \rightarrow 1$ ).

PROOF OF 1) $\rightarrow 2$ ). Assume that $\{\mathfrak{V}, T(a)\}$ fulfills i). Then for any $x \in \mathfrak{B}$ and for any positive number $\varepsilon$, there exists an element $y$ belonging to an invariant finite-dimensional subspace $\mathfrak{B}_{1}$ such that $\|x-y\|<\varepsilon / 3 M$, where $M$ is an upper bound of $\|T(a)\|$. Let $a_{a} \rightarrow a_{0}$. Since in $\mathfrak{B}_{1}$ the representation $T(a)$ is strongly continuous, for a suitable $\alpha_{0}$ we have $\left\|T\left(a_{\infty}\right) y-T\left(a_{0}\right) y\right\|<\varepsilon / 3$ when $\alpha>\alpha_{0}$. From this it follows immediately that $\left\|T\left(a_{a}\right) x-T\left(a_{0}\right) x\right\|<\varepsilon$ when $\alpha>\alpha_{0}$.

ProOF of 2$) \rightarrow 4$ ). Let $\{\mathfrak{B}, T(a)\}$ be strongly continuous. For any fixed $x \in \mathfrak{B}$, put $\mathfrak{B}_{1}=[T(a) x \mid a \in G]$ and $K=\{a ; T(a) x=x\}$; clearly $K$ is a closed subgroup of $G$. Introduce a new topology into the homogeneous space $G_{1}=G / K$ (left cosets) by making use of the metric such that

$$
\operatorname{dist}(\dot{a}, \dot{b})=\|T(a) x-T(b) x\|,
$$

where $\dot{a}$ means the coset containing $a$. We denote the topological space thus obtained by $G_{1}^{\prime}$. The natural mapping of $G$ onto $G_{1}^{\prime}$ being continuous and $G$ being compact, we find that $G_{1}^{\prime}$ is separable, whence we can choose a countable dense subset $\left\{\dot{a}_{n}\right\}$ from $G_{1}^{\prime}$. Then $\mathfrak{B}_{1}$ is spanned by the subspace formed by such elements as $\sum \alpha_{n} T\left(a_{n}\right) x$ with

12) This property of $T(a)$ is called separably-valued. When we assume merely the separability of $\left[T(a) x \mid a \epsilon G-N_{x}\right]$, where $N_{x}$ means a suitable null set, $T(a)$ is called almost separably-valued. 
rational coefficients $\alpha_{n}$.

PROOF of 4$) \rightarrow 3$ ). This is a consequence of a well-known lemma due to Pettis [11], which asserts that a weakly measurable $T(a) x$ becomes strongly measurable if and only if $T(a) x$ is almost separably. valued.

PROOF of 3$) \rightarrow 1$ ). Using the same notations as in Proposition A, we have only to show that $\widetilde{\subseteq}$, constructed in this case for $\{\mathfrak{B}, T(a)\}$, coincides with $\mathfrak{B}$, under the assumption of strong measurability. Now, as is readily verified by ii) of Proposition A, it is sufficient for this to prove $\widetilde{\subseteq} \subseteq \mathfrak{B}$; $\widetilde{\subseteq}$ being generated by all $F_{\xi, f}\left(\xi \in \mathfrak{B}, f \in \mathfrak{B}^{*}\right)$, this is furthermore reduced to show that $F_{\xi, f}$ is contained in $\mathfrak{B}$, because $\mathfrak{B}$ is closed in $\mathfrak{B}^{* *}$.

Since the function $x_{\xi, f}(a)$, defined on $G$ with the range $\mathfrak{B}$, such that

$$
x_{\xi, f}(a)=\overline{f(T(a) \xi)} T(a) \xi
$$

is strongly measurable and $\left\|x_{\xi, f}(a)\right\| \|$ is bounded, $x_{\xi, f}(a)$ is strongly integrable (in the sense of Bochner). Hence, putting

$$
x_{\xi, f}=\int x_{\xi, f}(a) d a,
$$

we have

$$
\begin{aligned}
F_{\xi, f}(g) & =\int g(T(a) \xi) \overline{f(T(a) \xi)} d a \\
& =g\left(\int \overline{f(T(a) \xi)} T(a) \xi d a\right) \\
& =g\left(x_{\xi, f}\right) \quad \text { for all } g \in \mathfrak{B}^{*} .
\end{aligned}
$$

This means $F_{\xi, f}=x_{\xi, f}$, as we wish to show. Thus all parts of Theorem 2 are completely proved.

As easy applications of Theorem 2 to the regular representation in a function space: $f(x) \rightarrow f(x a)$, we have the following well-known results.

Corollary 1 (Peter-Weyl). Denote by $R(G)$ the linear set generated by the coefficients of all finite-dimensional irreducible representations of $G$. Then $R(G)$ forms a dense linear set in $L^{p}(G)(1 \leqq p<\infty)$ and in $C(G)$ with respect to the proper topology in each space.

We mean by $C r\left(T^{n}\right)$ the family of all functions of the class $C^{r}$ on 
the $n$-dimensional torus $T^{n}$.

COROLlaRY 2. Let $f\left(x_{1}, \cdots, x_{n}\right) \in C^{r}\left(T^{n}\right)(r \leqq \infty)$. For a given $\varepsilon>0$ and for an integer $N$ such that $N \leqq r$, there exists a trigonometrical polynomial $P(x)$ with the properties:

$$
\left|D^{(k)} f\left(x_{1}, \cdots, x_{n}\right)-D^{(k)} P\left(x_{1}, \cdots, x_{n}\right)\right|<\varepsilon
$$

for $0 \leqq k \leqq N$, where $D^{(k)} f$ is the $k \cdot$ th derivative of $f$.

\section{$\S 5$. Structure of weakly continuous representations.}

We shall prove that any weakly continuous representation is expressed as a kind of a continuous direct sum of strongly continuous representations, each of which is realized as the regular representation in a function space. This aspect is very analogous to the situation that any $\mathrm{W}^{*}$-algebra (ring, according to a terminology of von Neumann) on a separable Hilbert space can be reduced to factors and is written as a direct integral of them, as is shown by von Neumann [9], Segal [12] and Godement [2].

THEOREM 3. Let $\{\Re, T(a)\}$ be a weakly continuous representation of $G$. Then we can find a locally compact space $\Omega$, and a non-trivial normed linear space $\Gamma_{\xi}$ corresponding to each point $\xi$ of $\Omega ; I_{\xi}$ is a subfamily of continuous functions on $G$ with a suitable norm, and is also anti-isomorphic to an invariant subspace of $\left\{\mathfrak{N}^{*}, T^{*}(a)\right\}$. Furthermore we have a linear mapping of $\mathfrak{l l}$ onto $\Gamma_{\xi}$ such that $x \rightarrow x_{\xi}(a)$. We denote by $x_{\xi}^{*}$ the element in $\mathfrak{i}^{*}$ corresponding to $x_{\xi}(a)$. The totality of $x_{\xi}(a)(\xi \in \Omega, x \in \mathfrak{l})$ can be considered as a linear space 4 , formed by such functions on $\Omega$ as taking values in $I^{\prime} \xi_{0}$ at $\xi=\xi_{0}$.

Those have the following properties:

1) The linear mapping

$$
x \rightarrow\left\{x_{\xi}(a)\right\}
$$

of $\mathfrak{R}$ onto $\Delta$ is univalent. This correspondence is denoted by $x \sim x_{\xi}(a)$.

2) $\left\|x_{\xi}(a)\right\|_{\xi} \leqq\|x\|$, where \|\|$_{\xi}$ means the norm in $\Gamma_{\xi}$.

3) $T(b) x \sim x_{\xi}(a b)$.

4) $\left\|x_{\xi}\left(a b_{\alpha}\right)-x_{\xi}\left(a b_{0}\right)\right\|_{\xi} \rightarrow 0$ as $b_{\alpha} \rightarrow b_{0}$, i.e. the right regular representation in $I_{\xi}$ is strongly continuous.

5) For fixed $a$ and $x, x_{\xi}(a)$ as a function of $\xi$ belongs to $L_{\infty}(\Omega)$, 
where $L_{\infty}(\Omega)$ means the function space formed by all continuous functions vanishing at infinity.

6) $\|T(a) x\|=\max _{\xi \in \Omega}\left|x_{\xi}(a)\right|$.

7) Let $x \sim x_{\xi}(a), y \sim y_{\xi}(a)$. Then

$$
y_{\xi}^{*}(x)=\int x_{\xi}(a) \overline{y_{\xi}(a)} d a .
$$

8) Let $x \sim x_{\xi}(a)$. If a sequence $\left\{\xi_{n}\right\}$ of $\Omega$ converges to $\xi$, then $x_{\xi_{n}}^{*}$ converges weakly to $x_{\xi_{0}}^{*}$.

Proof. Let $\Omega^{\prime}$ be the unit circle of $\Re^{*}$. Then, as is well-known, $\Omega^{\prime}$ is compact with respect to the weak topology. Thus, putting $\Omega$ for $\Omega^{\prime}-\{0\}$, we find that $\Omega$ is the locally compact space (the eliminated point 0 is considered to be at infinity). For any $x \in \mathfrak{R}$, we assign to each $f \in \Omega$ the continuous function on $G$ such that

$$
x_{f}(a)=f(T(a) x) .
$$

For a fixed $f \in \Omega$, let $\Gamma_{f}^{\prime}$ be the linear space consisting of all functions $x_{f}(a)$, where $x$ runs over $\mathfrak{N} ; I_{f}^{\prime}$ is clearly nontrivial. The mapping $x \rightarrow x_{f}^{\prime}(a)$ gives rise to a linear mapping of $\mathfrak{N}$ onto $I_{f}^{\prime}$.

In order to define a norm in $\Gamma_{f}^{\prime}$, we make use of Proposition B. Applying it to $\left\{\mathfrak{R}, T^{\prime}(a)\right\}$ in question, we see that $\widetilde{\mathfrak{L}}$ is an invariant closed subspaces of $\left\{\mathfrak{R}^{*}, T^{*}(a)\right\}$ which is generated by invariant subspaces $\mathfrak{R}_{f}^{*}\left(f \in \mathfrak{R}^{*}\right) ; \mathfrak{R}_{f}^{*}$ is formed by all elements such that

$$
\Phi_{f, x}(y)=\int f(T(a) y) \overline{f(T(a) x)} d a \quad(x \in \Re),
$$

as is remarked at the end of Proposition B. If $f(T(a) x) \equiv 0$, then of course $\Phi_{f, x} \equiv 0$; conversely $\Phi_{f, x} \equiv 0$ yields $\Phi_{f, x}(x)=0$, that is, $\int|f(T(a) x)|^{2} d a=0$ and so $f(T(a) x) \equiv 0$.

Therefore it is possible to introduce a norm in $\Gamma_{f}^{\prime}$ by

$$
\left\|x_{f}(a)\right\|_{f}=\left\|\Phi_{f, x}\right\| \text {. }
$$

As is seen from (6) and (7), the normed linear space $I_{f}^{\prime}$ whose constituents are $I_{f}^{\prime}$ and \|\|$_{f}$ is anti-isomorphic to $\Re_{f}^{*}$ by means of the correspondence $x_{f}(a) \longleftrightarrow \Phi_{f, x}$.

Now we shall prove that $\Omega, I_{f}^{\prime}$ and $x \rightarrow x_{f}(a)$ have the properties stated in the theorem. 
1) $x_{f}(a)=f(T(a) x) \equiv 0$ for all $f \in \Omega$, is equivalent to $x=0$.

2) By (7) and $\|f\| \leqq 1$, we may assume $\left\|\Phi_{f, x}\right\| \leqq\|x\|$, and so $\left\|x_{f}(a)\right\|_{f} \leqq\|x\|$.

3) From $x \sim x_{f}(a)$ and $x_{f}(a)=f(T(a) x)$, it results $T(b) x \sim x_{f}(a b)$.

4) Since by (7) $\Phi_{f, T(b) x}=T^{*}\left(b^{-1}\right) \Phi_{f, x}$, we have $\left\|x_{f}(a b)\right\|_{f}=$ $\left\|T^{*}\left(b^{-1}\right) \Phi_{f, x}\right\|$, and so

$$
\left\|x_{f}\left(a b_{\alpha}\right)-x_{f}\left(a b_{0}\right)\right\|_{f}=\left\|T^{*}\left(b_{\alpha}^{-1}\right) \Phi_{f, x}-T^{*}\left(b_{0}^{-1}\right) \Phi_{f, x}\right\|
$$

This tends to zero as $b_{a} \rightarrow b_{0}$, because $\left\{\Re_{f}^{*}, T^{*}(a)\right\}$ is strongly continuous.

5) This is easily seen by the definition of weak topology.

6) $\|T(a) x\|=\max _{\| f \mid \leq 1}|f(T(a) x)|=\max _{f \in \Omega}\left|x_{f}(a)\right|$.

7) By (6) and (7) this is clear.

8) Suppose that $f_{n} \rightarrow f_{0}$ (weakly); then $f_{n}(T(a) x) \rightarrow f_{0}(T(a) x)$ for each $a$, whence by (7) $\Phi_{f_{n}, x} \rightarrow \Phi_{f_{0}, x}$ (weakly).

This completes the proof.

REMARK. $\boldsymbol{\Omega}$ does not always satisfy the first axiom of countability. But it is known that, in the case when $\mathfrak{l}$ is a reflexive Banach space, $\boldsymbol{\Omega}$ is locally sequentially-compact.

Now it is desirable to take an available simple space as the base space $\Omega$ in the above theorem. Because we know by Theorem 2 the structure of strongly continuous representations, we arrive at

DEFINITION 2. Let $\{\mathfrak{N}, T(a)\}$ be a bounded algebraic representation. Assume that it is possible to find a strongly continuous representation $\{\subseteq, S(a)\}$ such that

1) There exists a continuous univalent linear mapping $\Psi$ of $\Re$ onto $\mathfrak{S}$.

2) $\Psi(T(a) x)=S(a) \Psi(x)$

Then $\{\Re, T(a)\}$ is called almost strongly-continuous.

THEOREM 4. For a given weakly continuous representation $\{\Re, T(a)\}$, assume that we can select suitable countable elements $f_{1}, f_{2}$, .. in $\mathfrak{S}^{*}$ such that $\left\{T^{*}(a) f_{i} ; a \in G, i=1,2, \cdots\right\}$ forms a total subset in $\mathfrak{R}^{*}{ }^{13)}$ Then $\{\mathfrak{R}, T(a)\}$ is almost strongly-continuous.

PROOF. Denote by $S^{\prime}$ the linear set consisting of all elements

13) A subset $\mathfrak{S}$ in the conjugate space of a normed linear space $\mathfrak{R}$ is called total, when, if $f(x)=0$ for all $f \in \mathfrak{S}$, then $x=0$. 
such that $\Phi_{x}=\left\{x_{f_{1}}(a), x_{f_{2}}(a), \cdots\right\}$, where $x_{f_{i}}(a)$ is defined by (6). This is a subset of a sequential space, whose coordinates are continuous functions on $G$. We introduce a norm in $\mathbb{S}^{\prime}$ by

$$
\left\|\Phi_{x}\right\|=\sum_{i=1}^{\infty} \frac{\left\|x_{f_{i}}(a)\right\|_{f_{i}}}{2^{i}} ;
$$

here we assume $\left\|f_{i}\right\|=1(i=1,2, \cdots)$ without losing generality.

Denoting by $\subseteq$ the normed linear space $\left\{\Im^{\prime} ;\|\|\right\}$, we define a mapping $\Psi$ of $\Re$ onto $S$ by $x \rightarrow \Phi_{x}$. Then $\Psi$ is obviously linear and continuous by 2) in Theorem 3. Now $\Phi_{x}=0$ means $\left\|x_{f_{i}}(a)\right\|_{f_{i}}=0$ for all $i$ and so $x_{f_{i}}(a) \equiv 0$, whence we have $f_{i}(T(a) x)=T^{*}(a) f_{i}(x)=0$ for $i=1,2, \cdots$ and for all $a \in G$. This, together with the assumption on $\left\{T^{*}(a) f_{i}\right\}$, yields $x=0$. Hence $\Psi$ is univalent.

Now the representation $\{\subseteq, S(a)\}$ with

$$
S(b) \Phi_{x}=\left\{x_{f_{1}}(a b), x_{f_{2}}(a b), \cdots\right\}
$$

is strongly continuous, onto which $\{\Re, T(a)\}$ is imbedded by $\Psi$. Hence $\{\Re, T(a)\}$ is almost strongly-continuous.

In $\$ 7$, we shall treat again almost strongly-continuous representations from a somewhat different viewpoint.

\section{\$. Relations between two notions of continuity.}

In this section we shall treat the relations between the weaklyand the strongly-continuous representations. ${ }^{14)}$

From Proposition A and Theorem 2 we have

THEOREM 5. In the case where the representation space $\mathfrak{R}$ is separable or reflexive, the weak continuity of $\{\mathfrak{N}, T(a)\}$ necessarily implies the strong continuity.

Let $\{\Re, T(a)\}$ be a bounded algebraic representation. Form an invariant closed subspace $\mathfrak{R}_{W}$ of $\mathfrak{R}$, consisting of all those elements $x$ for which $f(T(a) x)$ is continuous on $G$ for every $f \in \mathfrak{R}^{*} ;\left\{\mathfrak{N}_{W}, T(a)\right\}$ is the greatest weakly-continuous representation contained in $\{\Re, T(a)\}$, and is called the weakly continuous part of $\{\Re, T(a)\}$. The strongly

14) We could not succeed in obtaining an example of a weakly, but not strongly, continuous representation. 
continuous part $\left\{\Re_{S}, T(a)\right\}$ is defined similarly: $\mathfrak{N}_{S}$ is the invariant closed subspace of $\Re$, consisting of all those elements $x$ such that $x \in \Re_{W}$ and $[T(a) x \mid a \in G]$ is separable. It is clear that $\mathfrak{R}_{S} \subseteq \mathfrak{N}_{W} \subseteq \Re$.

We are now interested in studying a weakly continuous representation $\{\mathfrak{R}, T(a)\}$ with $\mathfrak{R} \neq \mathfrak{R}_{S}$.

Lemma 1. Using the same notations as in Proposition $A$, if $\widetilde{\subseteq} \subseteq \mathfrak{N}$, then $\{\subseteq, T(a)\}$ is strongly continuous.

ProOF. The assumption, combined with ii) of Proposition A, yields $\subseteq \subseteq \widetilde{\subseteq} ;\left\{\widetilde{\subseteq}, T^{* *}(a)\right\}$ being strongly continuous, $\{\subseteq, T(a)\}$ is of course strongly continuous.

Consequently, if a weakly continuous representation $\{\Re, T(a)\}$ with $\mathfrak{R} \neq \mathfrak{R}_{S}$ is given, applying Proposition A to $\{\mathfrak{R}, T(a)\}$ itself, we have $\widetilde{S} \cap\left(\mathfrak{N}^{* *}-\mathfrak{R}\right) \neq\{0\}$ and $\widetilde{S} \cap\left(\mathfrak{R}-\mathfrak{N}_{S}\right)=\{0\}$. Furthermore, by ii) of Proposition A, from $F(f)=0$ for all $F \in \widetilde{\subseteq}$ it results $f=0$. Thus we have shown

THEOREM 6. For any weakly continuous representation $\{\mathfrak{N}, T(a)\}$, the strongly continuous part $\mathfrak{N}_{S}^{* *}$ of $\left\{\mathfrak{N}^{* *}, T^{* *}(a)\right\}$ forms a total subspace. If $\mathfrak{R}_{S} \varsubsetneqq \mathfrak{R}$, then $\mathfrak{R}_{S} \subsetneq \mathfrak{R}_{S}^{* *}$.

COROLlARY. Let $\{\mathfrak{N}, T(a)\}$ be a bounded algebraic representation. Consider a closed subspace $\mathfrak{N}_{c}^{* *}$ of $\mathfrak{R}^{* *}$, formed by all elements $F$ such that $F\left(T^{*}(a) f\right)$ is a continuous function on $G$ for every $f \in \mathfrak{R}^{*}$. If $\mathfrak{P}_{C}^{* *}=\mathfrak{N}$, then $\{\mathfrak{R}, T(a)\}$ is strongly continuous.

The above discussions may be used to obtain a remarkable, but somewhat pathological property of a weakly continuous B-representation $\{\mathfrak{B}, T(a)\}$ with $\mathfrak{B} \neq \mathfrak{B}_{S}$. To see this some preliminary considerations are necessary.

Let $\mathfrak{B}$ be a non-reflexive Banach space. A theorem due to Milman asserts that a closed subspace of a reflexive Banach space is also reflexive, whence, repeating the process to construct $\mathfrak{B}^{* *}$ from $\mathfrak{B}$, we get

$$
\mathfrak{B}^{(0)} \subsetneq \mathfrak{B}^{(1)} \subsetneq \cdots \subsetneq \mathfrak{B}^{(n)} \subsetneq \cdots,
$$

where $\mathfrak{B}^{(0)}=\mathfrak{B}$ and $\mathfrak{B}^{(n+1)}=\mathfrak{B}^{(n) * *}$. This process is further carried on; by transfinite induction we shall define a Banach space $\mathfrak{B}^{(\alpha)}$ for any ordinal number $\alpha$. Suppose that, for an ordinal number $\alpha_{0}$, we have already defined $\mathfrak{B}^{(\alpha)}$ for $\alpha<\alpha_{0}$, with the property: $\mathfrak{B}^{(\alpha)} \subseteq \mathfrak{B}^{(\beta)}$ for $\alpha<\beta$. If $\alpha_{0}$ has an ordinal number $\gamma$ which is immediately before $\alpha_{0}$, 
we define $\mathfrak{B}^{\left(\alpha_{0}\right)}$ as $\mathfrak{B}^{(\gamma) * *}$. If $\alpha_{0}$ is a limit ordinal number, first put $\mathfrak{Q}^{\left(\alpha_{0}\right)}=\bigcup_{\alpha<\alpha_{0}} \mathfrak{R}_{\alpha}$; then $\mathfrak{Q}^{\left(\alpha_{0}\right)}$ becomes in a natural manner a normed linear space. Now we define $\mathfrak{B}^{\left(\alpha_{0}\right)}$ as a Banach space obtained by completion of $\mathfrak{L}^{\left(\alpha_{0}\right)}$. Thus we have obtained $\mathfrak{B}^{(\alpha)}$ for any $\alpha$, such that $\mathfrak{B}^{(\alpha)} \subseteq \mathfrak{B}^{(\beta)}$. for $\alpha<\beta$.

Assume that a weakly continuous B-representation $\{\mathfrak{B}, T(a)\}$ is given. Then we can define an operator $T^{(\alpha)}(a)$ on $\mathfrak{B}^{(\alpha)}$ in an obvious manner ; $\left\{\mathfrak{B}^{(\alpha)}, T^{(\alpha)}(a)\right\}$ is a bounded algebraic B-representation of $G$. We denote by $\left\{\mathfrak{B}_{W}^{(\alpha)}, T^{(\alpha)}(a)\right\}$ and $\left\{\mathfrak{B}_{S}^{(\alpha)}, T^{(\alpha)}(a)\right\}$ the weakly and the strongly continuous part of $\left\{\mathfrak{B}^{(\alpha)}, T^{(\alpha)}(a)\right\}$, respectively. Then from Proposition A and Lemma 1 we have

THEOREM 7. Let $\{\mathfrak{B}, T(a)\}$ be a weakly continuous B-representation such that $\mathfrak{B} \neq \mathfrak{B}_{S}$. Then $\mathfrak{B}_{S}^{(\alpha)} \subseteq \mathfrak{B}_{S}^{(\beta)}$ and $\mathfrak{B}_{W}^{(\alpha)} \subseteq \mathfrak{B}_{W}^{(\beta)}$ for any $\alpha<\beta$.

So far we have mainly concerned with the relationship between $\{\Re, T(a)\}$ and $\left\{\Re^{* *}, T^{* *}(a)\right\}$. From now on we shall consider the properties of $\left\{\Re^{*}, T^{*}(a)\right\}$ induced from a given representation $\{\mathfrak{N}, T(a)\}$. If the representation space is reflexive, the situation is very clear, that is, the strong continuity of $\{\mathfrak{B}, T(a)\}$ implies the strong continuity of $\left\{\mathfrak{B}^{*}, T^{*}(a)\right\}$ and vice versa.

DEFINITION 3. A bounded algebraic representation $\{\mathfrak{R}, T(a)\}$ is. called almost weakly.continuous when $\mathfrak{R}_{C}^{*}$ is a total set in $\mathfrak{R}^{*}{ }^{15}$ )

From Proposition B and this definition we obtain:

THEOREM 8. Let $\{\Re, T(a)\}$ be an almost weakly-continuous representation. Then the strongly continuous part $\mathfrak{R}_{S}^{*}$ of $\left\{\mathfrak{N}^{*}, T^{*}(a)\right\}$ is total in $\mathfrak{N}^{*}$.

We shall give an example of $\{\mathfrak{R}, T(a)\}$ such that $\mathfrak{R}_{S}^{*}$ is total in $\mathfrak{R}^{*}$. Let $\{\Re, T(a)\}$ be the regular representation induced in $L^{1}(G)$; then $\left\{\Re_{S}^{*}, T^{*}(a)\right\}$ is equivalent to the regular anti-representation induced in $C(G)$, and $C(G)$, regarded as a subspace of $L^{\infty}(G)$, is total in $L^{\infty}(G)$.

As a result of the above theorem we have

COROLLARY. If a weakly continuous representation $\{\Re, T(a)\}$ is regarded as the conjugate representation of a certain representation, ${ }^{16)}$ then $\{\Re, T(a)\}$ contains the strongly continuous part as a total subset.

15) As to the definition of $\mathfrak{N}_{C}^{*}$, see p.232.

16) Namely, we can find an algebraic anti-representation $\{\subseteq, S(a)\}$ such that $\mathfrak{R}=\mathfrak{S}^{*}$, $T(a)=S^{*}(a) . \quad\{\mathfrak{R}, T(a)\}$ is called the conjugate representation of $\{\circlearrowleft, S(a)\}$. 
From this corollary a problem arises: Does there exist a weakly continuous representation such that its strongly continuous part is $\{0\}$ ? The author has no idea to approach this problem.

Now let $\left\{\Re^{*}, T^{*}(a)\right\}$ be the conjugate representation of a given weakly continuous representation $\{\Re, T(a)\}$. Concerning the formal structure of $\left\{\mathfrak{N}^{*}, T^{*}(a)\right\}$, we can obtain the corresponding result to Theorem 3 by the use of Proposition A. The essential distinction arising here lies in the fact that in this case it is assured that the base space $\Omega$ is only completely-regular instead of locally-compactness, contrary to Theorem 3. Since this assertion can be easily formulated, we omit the exact formulation.

\section{§7. Mesurable B-representations.}

Concerning unitary representations, we know that, whenever the representation space is separable, the weak measurability of a representation necessarily leads to the strong continuity. We proceed as before to reduce our discussions on measurable B-representations to this result of the unitary representation. For that purpose the following lemma is useful, the devices of whose proof are due to E. Hopf [4].

Lemma 2. Let $\{\mathfrak{B}, T(a)\}$ be strongly measurable. Then

$$
\int\left|f_{0}\left(T(a) x_{0}\right)\right|^{2} d a=0
$$

implies $f_{0}\left(x_{0}\right)=0$.

Proof. Owing to a lemma of Pettis, for a null set $M_{x_{0}}, \mathfrak{S}_{x_{0}}=$ $\left[T(a) x_{0} \mid a \notin M_{x_{0}}\right]$ is separable, where we may assume $e \notin M_{x_{0}}$. Let $x_{1}$, $x_{2}, \cdots$ be a countable dense subset of $\mathfrak{B}_{x_{0}}$. Now consider the closed subspace of 23 which consists of all elements $x$ such that $f_{0}(T(a) x)=0$ holds for $a \notin N_{x}$, where $N_{x}$ means a suitable null set. Clearly this subspace contains all $T(a) x_{0}$ for $a \in G$ and thus a fortiori $\mathfrak{B}_{x_{0}}$. Putting $N=\bigcup_{n=1}^{\infty} N_{x_{n}}$, we see that $\Re$ is a null set and that $f_{0}(T(a) x)=0$ for $a \notin N$, irrespective of $x \in \mathfrak{B}_{x_{0}}$. Hence, putting $x_{0}$ for $x$ in this equality and then replacing $x_{0}$ by $T(b) x_{0}$ for any $b \notin M_{x_{0}}$, we have $f_{0}\left(T(a b) x_{0}\right)=0$ for $a \notin N$. On the other hand, it follows immediately from the boundedness of Haar measure that there exists an element $c$ such that $c \notin N$, 
$c^{-1} \notin M_{x_{0}}$. Hence we have $f_{0}\left(x_{0}\right)=f_{0}\left(T\left(c c^{-1}\right) x_{0}\right)=0$, as we wished to prove. THEOREM 9. If $\mathfrak{B}^{*}$ is separable, the weakly measurable B-representation $\{\mathfrak{B}, T(a)\}$ is strongly continuous.

PROOF. This theorem holds for a finite-dimensional case. Applying the consideration similar to the one used in the proof of Theorem 2, we see that it suffices to show the complete decomposability of $\{\mathfrak{B}, T(a)\}$.

We first prove:

a) Let $\left\{\mathfrak{S}_{\xi}, U(a)\right\}$ be the algebraic unitary representation which is obtained from $\{\mathfrak{B}, T(a)\}$ by the procedures used in Proposition A (as is easily seen, this is possible). Then $\left\{\mathfrak{S}_{\xi}, U(a)\right\}$ is a (continuous) unitary representation.

b) If $(f, f)_{\xi}=\int|f(T(a) \xi)|^{2} d a=0$, then $f(\xi)=0$.

PROOF OF a). It is clear that the separability of $\mathfrak{B}^{*}$ implies that of $\mathfrak{S}_{\xi}$; besides

$$
(U(a) \dot{f}, \dot{g})_{\xi}=\int f\left(T\left(a^{-1} b\right) \xi\right) g(T(b) \xi) d b
$$

is measurable. Hence $\left\{\mathfrak{h}_{\xi}, U(a)\right\}$ is a continuous representation, according to a known result for the unitary representation.

PROOF OF b). Since $\mathfrak{B}$ is separable because of the separability of $\mathfrak{B}^{*},\{\mathfrak{B}, T(a)\}$ is strongly measurable and so Lemma 2 can be applied.

Now, as is easily verified, the above two assertions enable us to carry over the proof of Proposition A and the proof of iii) $\rightarrow$ i) of Theorem 2 to $\{\mathfrak{B}, T(a)\}$ in question. Thus, if we follow this, we get the complete decomposability of $\{\mathfrak{B}, T(a)\}$, which completes the proof.

In the above theorem the separability of $\mathfrak{B}^{*}$ is assumed. The author cannot determine whether this assumption may be replaced by a more weakened one such as the separability of $\mathfrak{B}$. However we shall show later that this is the case when $\{\mathfrak{B}, T(a)\}$ is the conjugate representation of some representation.

DEFINITION 4. Let $\mathfrak{B}$ be a Banach space. If a subset $\mathfrak{I}$ of $\mathfrak{B}^{*}$ satisfies the following condition, $\mathfrak{I}$ is called strictly total:

$$
\sup _{f \in \mathfrak{S}, f \neq 0} \frac{|f(x)|}{\|f\|}=\|x\| \quad \text { for all } x \in \mathfrak{B} .
$$


Obviously a strictly total set is total. We have examples of such sets: 1) $\mathfrak{B}$, as a subspace of $\left.\mathfrak{B}^{* *}, 2\right) C(G)$, as a subspace of $L^{\infty}(G)$, which is conjugate to $\left.L^{1}(G), 3\right)$ The subspace in the conjugate space of $C(G)$, consistng of all Radon measures $\{d \mu\}$ such that $d \mu=f(x) d x$ $(f(x) \in C(G))$.

DEFINITION 5. A bounded algebraic B-represeptation $\{\mathfrak{B}, T(a)\}$ is called s-almost weakly.continuous in the case where $\mathfrak{B}_{C}^{*}$ is strictly total.

In order to make the position of the following theorem clear, we first give a general property of almost strongly-continuous representations.

PROPOSITION 4. An almost strongly-continuous representation $\{\Re, T(a)\}$ is necessarily almost weakly-continuous.

PROof. By Definition $2\{\Re, T(a)\}$ is continuously imbedded onto a strongly continuous representation $\{\Theta, S(a)\}$. Denote by $\Psi$ this linear univalent mapping of $\Re$ onto $\subseteq$, and by $\Psi^{*}$ its adjoint mapping of $\mathbb{S}^{*}$ into $\mathfrak{A}^{*}$. Observe that, for every $F \in \mathbb{S}^{*} \Psi^{*}(F)(T(a) x)$ is continuous for each $x \in \mathfrak{R}$, i. e. $\Psi^{*}\left(\subseteq^{*}\right) \subseteq \mathfrak{R}_{C}^{*}$. This is immediately seen from the strong continuity of $\{\subseteq, S(a)\}$ and

$$
\begin{aligned}
\Psi^{*}(F)(T(a) x) & =F(\Psi(T(a) x)) & & \text { (from the definition of } \left.\Psi^{*}\right) \\
& =F(S(a) \Psi(x)) & & \text { (from Definition 2). }
\end{aligned}
$$

Now if $\Psi^{*}(F)(x)=0$ for all $F \in \mathbb{S}^{*}$, then $F(\Psi(x))=0$, and so $\Psi(x)=0$, $x=0$. This means that $\Psi^{*}\left(\Im^{*}\right)$ is total, and thus $\mathfrak{R}_{C}^{*}$ is total. This completes the proof.

THEOREM 10. Assume that $\{\mathfrak{B}, T(a)\}$ is a strongly measurable $B$-representation. If $\{\mathfrak{B}, T(a)\}$ is almost weakly-continuous, it contains a dense invarirnt subspace (5 such that $\{\mathfrak{C}, T(a)\}$ is an almost strongly. continuous representation. If $\{\mathfrak{B}, T(a)\}$ is further assumed to be $s$ almost weakly-continuous, $\{\mathfrak{B}, T(a)\}$ becomes strongly continuous.

PROOF OF THE First AsSERTion. Consider the total closed subspace $\mathfrak{B}_{C}^{*}$ stated in Definition 3. For $f, g \in \mathfrak{B}_{C}^{*}$, we define an inner product by

$$
(f, g)_{\xi}=\int f(T(a) \xi) \overline{g(T(a) \xi)} d a .
$$

Then, by the repetition of the same arguments as in Proposition A, 
we can conclude that there exists a closed subspace $\widetilde{\widetilde{S}}$ of $\left(\mathfrak{B}_{C}^{*}\right)^{*}$ such

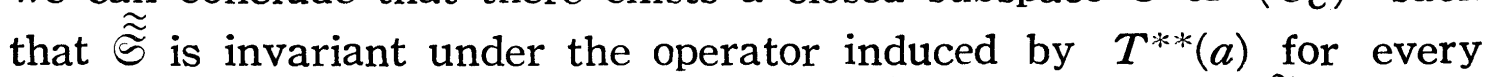
$a \in G$ (this operator is denoted by the same notation) and $\left\{\widetilde{\widetilde{S}}, T^{* *}(a)\right\}$ is strongly continuous. The elements $F_{\xi, g}$ of $\widetilde{\Xi}$ corresponding to $\xi \in \mathfrak{B}$, $g \in \mathfrak{B}_{C}^{*}$, that is, defined by

$$
F_{\xi, g}(f)=\int f(T(a) \xi) \overline{g(T(a) \xi)} d a \quad\left(f \in \mathfrak{B}_{C}^{*}\right)
$$

generate a dense invariant subspace $\mathfrak{S}$ in $\widetilde{\widetilde{S}}$.

Put

$$
x_{\xi, g}=\int \overline{g(T(a) \xi)} T(a) \xi d a .
$$

From the assumption this Bochner integral exists. (8) and (9) yield

$$
f\left(x_{\xi, g}\right)=F_{\xi, g}(f) \quad \text { for all } f \in \mathfrak{B}_{C}^{*} \text {. }
$$

Since $\mathfrak{B}_{C}^{*}$ is total, $x_{\xi, g}$ is uniquely determined by the above relation. From this, putting $\mathfrak{F}$ for the subspace generated by $x_{\xi, g}\left(\xi \in \mathfrak{B}, g \in \mathfrak{B}_{C}^{*}\right)$, we know that $\mathfrak{E}$ is an invariant subspace of $\{\mathfrak{B}, T(a)\}$, and the mapping $\Psi$ defined by $\Psi\left(x_{\xi, g}\right)=F_{\xi, g}$ gives rise to a univalent linear mapping of $\mathfrak{S}$ onto $\mathfrak{S}$. Now

$$
\left\|\Psi\left(x_{\xi, g}\right)\right\|=\sup _{f \in \mathfrak{B}^{*} C,\|f\|=1}\left|f\left(x_{\xi, g}\right)\right| \leqq\left\|x_{\xi, g}\right\|,
$$

whence $\Psi$ is continuous. Besides clearly

$$
\Psi(T(a) x)=T^{* *}(a) \Psi(x) .
$$

Thus $\{\mathfrak{b}, T(a)\}$ is almost strongly-continuous.

Consequently, if we can show that $\mathfrak{S}$ is dense in $\mathfrak{B}$, the proof will be completed. This is done as follows.

Take $f \in \mathfrak{B}^{*}$ such that $f(x)=0$ for all $x \in \mathfrak{F}$. Then this means $f\left(x_{\xi, g}\right)=0$ for any $\xi \in \mathfrak{B}, g \in \mathfrak{B}^{*}$, whence we have by (9)

$$
\int \overline{g(T(a) \xi)} f(T(a) \xi) d a=0 .
$$

This is written as

$$
\overline{g\left(\int \overline{f(T(a) \xi)} T(a) \xi d a\right)}=0 \quad \text { for all } \xi \in \mathfrak{B}, g \in \mathfrak{B}_{C}^{*} .
$$


But $\mathfrak{B}_{C}^{*}$ being total, it results

$$
\int \overline{f(T(a) \xi)} T(a) \xi d a=0 \quad \text { for all } \xi \in \mathfrak{B} .
$$

Here applying $f$, we have

$$
\int|f(T(a) \xi)|^{2} d a=0 \quad \text { for all } \xi \in \mathfrak{B},
$$

whence by Lemma 2 we obtain $f=0$. This completes the proof.

Proof of the Second Assertion. Suppose that $\{\mathfrak{B}, T(a)\}$ is strongly measurable and s-almost weakly-continuous. When we apply the above proof to $\{\mathfrak{B}, T(a)\}$, by Definition 4 we have the equality sign in (10), and so $\mathfrak{S}$ may be identified with $\mathfrak{E}$, from which the strong continuity of $\{\mathfrak{B}, T(a)\}$ follows.

REMARK. The second part of this theorem is a strengthened form of Theorem 2, 3) $\rightarrow 2$ ).

Corollary. Suppose that $\{\mathfrak{B}, T(a)\}$ is a weakly-continuous $B$ representation and $\left\{\mathfrak{B}^{*}, T^{*}(a)\right\}$ is strongly measurable. Then $\left\{\mathfrak{B}^{*}\right.$, $\left.T^{*}(a)\right\}$ is strongly continuous.

This follows from the fact that $\left\{\mathfrak{B}^{*}, T^{*}(a)\right\}$ is s-almost weaklycontinuous because $\mathfrak{B}$ is strictly total in $\mathfrak{B}^{* *}$.

Now, in order to derive some consequences from this corollary, we need a following result which is a slight generalization of Pettis' lemma [11].

LEMMA 3. Let $\mathfrak{B}$ be a Banach space and $\odot$ be a strictly total subspace in $\mathfrak{B}^{*}$. Let $x(\alpha)$ be a mapping of measure space $(\Omega, B, m)$ into $\mathfrak{B}$ such that $x(\alpha)$ is almost separably-valued. If $f(x(\alpha))$ is a measurable function on $\Omega$ for every $f \in \mathbb{S}$, then $x(\alpha)$ is strongly measurable.

PROOF ${ }^{17)}$ We shall first prove the measurability of $\|x(\alpha)\|$. Without losing generality, we may assume $x(\Omega)=\{x(\alpha) ; \alpha \in \Omega\}$ is separable. Let $\left\{x_{n}\right\}=\left\{x\left(\alpha_{n}\right)\right\}$ be a countable dense set in $x(\Omega)$. $\subseteq$, being strictly total, contains elements $\left\{f_{n, m}\right\}$ such that

17) See Hille [3], p. 36. 


$$
\begin{gathered}
\left\|f_{n, m}\right\|=1, \\
\left\|x_{n}\right\| \geqq\left|f_{n, m}\left(x_{n}\right)\right| \geqq\left\|x_{n}\right\|-\frac{1}{m} .
\end{gathered}
$$

Put $\phi_{m}(\alpha)=\sup _{n}\left|f_{n, m}(x(\alpha))\right|$. Then by the measurability of $f_{n, m}(x(\alpha))$ we see that $\boldsymbol{\varphi}_{m}(\alpha)$ is also measurable. For any $x(\alpha)$ and for any given $\varepsilon>0$ there exists an $x_{n}$ such that $\left\|x(\alpha)-x_{n}\right\|<\varepsilon$, whence we have

$$
\begin{aligned}
\varphi_{m}(\alpha) & \geq\left|f_{n, m}(x(\alpha))\right| \\
& \geq\left|f_{n, m}\left(x_{n}\right)\right|-\left|f_{n, m}\left(x(\alpha)-x_{n}\right)\right| \\
& \geq|| x(\alpha)||-\frac{1}{m}-2 \varepsilon,
\end{aligned}
$$

and so

$$
\boldsymbol{\varphi}_{m}(\alpha)+\frac{1}{m} \geqq\|x(\alpha)\| \geqq \boldsymbol{\varphi}_{m}(\alpha)
$$

Hence we have $\sup _{m} \varphi_{m}(\alpha)=\|x(\alpha)\|$, which means the measurability of $\|x(\alpha)\|$. Now the strong measurability of $x(\alpha)$ follows immediately from this result by the use of the familiar argument.

We can now show that there exist some remarkable relations between $\{\mathfrak{B}, T(a)\}$ and $\left\{\mathfrak{B}^{*}, T^{*}(a)\right\}$ under the assumption on the separability of $\mathfrak{B}^{*}$.

THEOREM 11. Let $\{\mathfrak{B}, T(a)\}$ be a weakly measurable B-representation. If $\mathfrak{B}^{*}$ is separable, then $\left\{\mathfrak{B}^{*}, T^{*}(a)\right\}$ is strongly continuous.

Proof. From Lemma 3 it results that $\left\{\mathfrak{B}^{*}, T^{*}(a)\right\}$ is strongly measurable since $\mathfrak{B}$ is strictly total in $\mathfrak{B}^{\mathfrak{k}^{*}}$; on the other hand, Theorem 9 shows the strong continuity of $\{\mathfrak{B}, T(a)\}$ under our assumption. Thus Corollary of Theorem 10 can be applied, which yields the desired result.

COROLLARY 1. If $\mathfrak{B}^{*}$ is separable, the weak measurability of $\{\mathfrak{B}$, $T(a)\}$ implies the strong continuities of $\left\{\mathfrak{L}^{*}, T(a)\right\}$ and $\left\{\mathfrak{B}^{*}, T^{*}(a)\right\}$.

The following corollary should be compared with Theorem 9.

COROLlARY 2. Assume that a weakly measurable B-representation $\{\mathfrak{B}, T(a)\}$ is the conjugate representation of a certain representation. Then $\{\mathfrak{B}, T(a)\}$ is strongly continuous whenever $\mathfrak{B}$ is separable.

THEOREM 12. If $\mathfrak{B}^{*}$ is separable, the strong continuity of $\{\mathfrak{B}$, 
$T(a)\}$ means the same property of $\left\{\mathfrak{B}^{*}, T^{*}(a)\right\}$ and vice versa.

PROOF. If $\{\mathfrak{B}, T(a)\}$ is strongly continuous, it is of course weakly measurable, so that by Theorem $11\left\{\mathfrak{B}^{*}, T^{*}(a)\right\}$ is strongly continuous. The converse part follows from Theorem 5 , since $\mathfrak{B}$ is separable.

\section{University of Tokyo.}

\section{Bibliography}

[1] R. Godement, Les fonctions de type positif et la théorie des groupes, Trans. Am. Math. Soc. 63 (1948), 1-84.

[2] R. Godement, Sur la théorie des représentations unitaires, Ann. Math. 53 (1951), 68-124.

[3] E. Hille, Functional analysis and semi-groups, New York (1948).

[4] E. Hopf, Ergodentheorie, Berlin (1947).

[5] A. Hurevitsch, Unitary representations in Hilbert space of a compact group, Rec. Math. 13 [55] (1943), 79-86.

[6] S. Itô, On irreducible unitary representations of a compact group and an abelian group (in Japanese), Sugaku 5 (1954), 226-228.

[7] I. Kaplansky, Primary ideals in group algebras, Proc. Nat. Acad. Sci. U. S. A. 35 (1949), 133-136.

[8] S. Murakami, On unitary representations of compact groups, Kodai Math. Sem. Rep. 3 (1951), 15-18.

[9] J. v. Neumann, Reduction theory, Ann. Math. 50 (1949), 401-485.

[10] F. Peter and H. Weyl, Die Vollständigkeit der primitiven Darstellungen einer geschlossen kontinuierlichen Gruppe. Math. Ann. 17 (1927), 737-755.

[11] B. J. Pettis, On integration in vector spaces, Trans. Am. Math. Soc. 44 (1938), 277-304.

[12] I. E. Segal, Decompositions of operator algebras I., Memoirs Am. Math. Soc. (1951).

[13] A. Weil, L'intégration dans les groupes topolgiques et ses applications, Act. Sci. Ind., Paris (1940).

[14] E. Wigner, On unitary representations of the inhomogeneous Lorenz group, Ann. Math. 40 (1939), 149-204. 